\title{
Factors associated with female high-risk drinking in a rural and urban South African site
}

Oluwayemisi A Ojo, Goedele Louwagie, Neo Morojele, Kirstie Rendall-Mkosi, Leslie London, Steve Olorunju, Adlai Davids

Objective. To assess and compare the extent of high-risk drinking and factors associated with high-risk drinking in the adult female population of a rural and an urban region in South Africa.

Design. Cross-sectional household survey using multistage sampling methods.

Setting. A rural wine farming area of the Western Cape and an urban site in Gauteng.

Subjects. Women of reproductive age (18 - 44 years).

Outcome measures. The extent of risky alcohol consumption measured using the Alcohol Use Disorders Identification Test (AUDIT) scale which categorises current drinkers into highand low-risk drinkers.

Results. Twenty-seven per cent (166/606) and 46\% (188/412) of the women interviewed in Gauteng and the Western Cape respectively were current drinkers. In turn, 20\% (33/166) of the Gauteng current drinkers and $68 \%(128 / 188)$ of the Western Cape current drinkers were classified as high-risk drinkers. Multivariate analysis indicated that employed people in Gauteng were less likely to be high-risk drinkers (odds ratio (OR) 0.3, 95\% confidence interval (CI) 0.1 - 0.8 ). Living in a household that never/seldom went hungry was inversely associated with risky drinking for both sites (OR 0.3 , 95\% CI 0.1 - 0.9 for Gauteng and OR 0.2, 95\% CI $0.3-1.2$ for the Western Cape). Current smokers (Western Cape) (OR 7.6, $95 \%$ CI 3.1 - 18.9) and respondents with an alcohol problem in one or more family members (both sites) (OR 6.0, 95\% CI 2.3 - 15.7 and OR $3.1,95 \%$ CI 1.5 - 6.4) were more likely to be high-risk drinkers.

Conclusions. High-risk drinking by women is a major problem, especially in the Western Cape. Targeted interventions are needed for women with alcohol problems in the family setting, lower socio-economic status, and concurrent substance abuse.

S Afr Med J 2010; 100: 180-182.
Excessive alcohol consumption is responsible for many health, psychological, sexual, social and economic problems. In South Africa in $2002,{ }^{1} 7 \%$ of disability-adjusted life-years (DALYS) and $7.1 \%$ of deaths were attributable to alcohol.

Alcohol consumption in South Africa has a particular historical context. There was an indigenous tradition of home brewing but, during the apartheid era, access to alcohol by the black majority was prohibited or restricted, which led to the proliferation of homebrews and widespread small-scale outlets called shebeens. ${ }^{2}$ Another unique feature in South Africa was the dop system, whereby farm workers were paid part of their wages in the form of alcohol - invariably wine. Although

School for Health Systems and Public Health, University of Pretoria

Oluwayemisi A Ojo, BPharm, MPH

Goedele Louwagie, MB ChB, MMed (PHM), FCPHM (SA)

Kirstie Rendall-Mkosi, BSc (OT), MPH

Alcohol and Drug Abuse Research Unit, Medical Research Council, Pretoria Neo Morojele, MSc, PhD

Biostatistics Unit, Medical Research Council, Pretoria Steve Olorunju, MSc, $\mathrm{PhD}$

School of Public Health and Family Medicine, University of Cape Town Leslie London, MB ChB, MMed PHM, DOH, BSc Hons (Epi)

Surveys, Analyses, Modelling and Mapping Division, Human Sciences Research Council, Pretoria

Adlai Davids, BA Hons, MSc

Corresponding author: G Louwagie (goedele.louwagie@up.ac.za) illegal since 1961, the dop system was nevertheless practised on some farms until quite recently. ${ }^{2}$

One consequence of high alcohol consumption among women is maternal drinking, leading to fetal alcohol syndrome (FAS), with reported rates for Grade 1 children of $6.8-8.9 \%{ }^{3}$ and $2.7 \%{ }^{4}$ in high-risk areas of the Western Cape and Gauteng, respectively.

Our study was part of a larger survey investigating the risks for FAS, in order to plan preventive interventions. We aimed to compare the extent of, and to assess factors associated with, high-risk drinking in women of reproductive age in a rural and an urban South African site.

Gauteng and Western Cape sites that differ in urbanisation status and alcohol consumption tradition were purposely selected. The Gauteng site comprised adjacent, historically black, coloured, and white areas in the Tshwane Municipality. The Western Cape site was a rural, predominantly commercial and agricultural region, producing wheat, vegetables, fruit and wine. Many workers live in dwellings belonging to the owners and are in extremely dependent relationships with their employers. Others live in local towns and are employed seasonally on farms, with poor job security and benefits.

\section{Methods}

A cross-sectional household survey was undertaken in 2006, including females aged 18 - 44 years. For Gauteng, a proportional sampling of enumeration areas (EAs) was obtained from the EA listing for the area, based on the 2001 population figures. Ten households were randomly selected from each of 82 selected EAs, using aerial maps, with 1 


\section{Original ARTICLES}

respondent selected randomly within each household. For the Western Cape, proportional sampling was conducted from 1450 listed farms, stratified across 3 municipal areas. All women in the eligible age categories on each farm were recruited. A total of 606 women were interviewed in Gauteng (response rate of 74\%) and 412 in the Western Cape (response rate of $83 \%$ ).

A structured questionnaire, translated into the 5 most commonly used languages of the research population, was used to collect demographic data on reproductive health factors and substance and contraceptive use. Trained fieldworkers conducted face-to-face interviews at the respondents' homes, after obtaining written informed consent.

Current drinkers were identified by a positive answer to the question 'Do you still take a drink with alcohol sometimes?' The extent of risky alcohol consumption was measured using the 10-item Alcohol Use Disorders Identification Test (AUDIT). ${ }^{5}$ Answers have a score ranging from 0 - 4. An individual's AUDIT score is the sum of the scores for each question; a score of 7 or less indicates low-risk drinking, while scores of 8 - 40 indicate high-risk drinking.

Questions relating to drinking patterns included: age of onset of alcohol consumption, having one or more family members with an alcohol problem, and types of beverage usually drunk. Smoking variables included current smoking and having ever experimented with cigarettes. Proxies for socio-economic variables measured were: how often the household goes hungry; employment status; highest level of education; and the presence of 8 selected amenities in the household.

Descriptive statistics were estimated for the entire sample including non-drinkers. Simple logistic regression, adjusted for EAs (Gauteng) or farm (Western Cape) clustering, was followed by multiple logistic regression to determine factors associated with high-risk drinking in current drinkers. Variables associated with high-risk drinking from previous studies were entered into the initial model (footnote, Table I).

\section{Results}

In Gauteng, $81 \%$ of the respondents were black and $42 \%$ were currently employed. In the Western Cape, $90 \%$ of the respondents were coloured and 80\% were employed. 166/606 $(27 \%)$ and $188 / 412(46 \%)$ of the respondents in Gauteng and the Western Cape, respectively, reported current drinking. Of the 166 current drinkers in Gauteng, 33 (20\%) were high-risk drinkers, compared with 120 (64\%) out of 188 in the Western Cape. Levels of drinking differed significantly among the sites (Fig. 1).

Beer and papsak wine were most commonly drunk in the Western Cape, while women in Gauteng were more likely to drink ciders and coolers. However, high-risk drinking was significantly associated with home brew and beer in Gauteng, and bottled and papsak wine in the Western Cape.

In univariate analysis, a higher educational level, the household never/seldom going hungry, and employment were inversely associated with high-risk drinking in Gauteng. Having 5 or more listed household amenities was inversely associated with high-risk drinking at both sites. For Gauteng, onset of alcohol consumption after the age of 18 was protective of high-risk drinking. Being a current smoker and having experimented with cigarettes were associated with high-risk drinking in the Western Cape. Respondents with one or more family members with an alcohol problem were more likely to be high-risk drinkers in both sites.

In multiple logistic regression modelling, the following socioeconomic measures were inversely associated with high-risk drinking: current employment (Gauteng), possessing 5 or more household amenities (Western Cape) and living in a household that never/seldom goes hungry (both sites). Current smokers

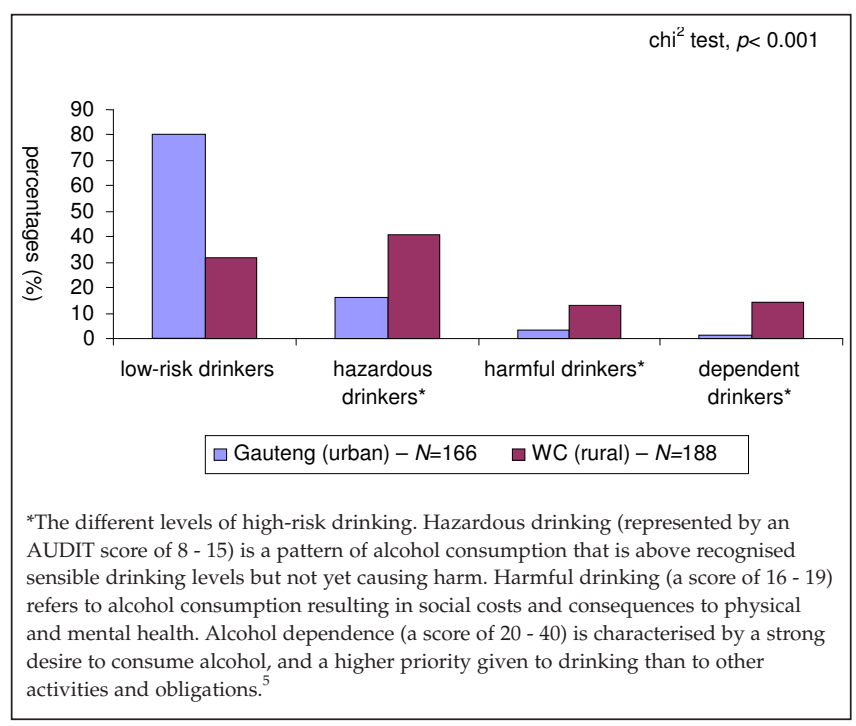

Fig. 1. Level of drinking in current drinkers using AUDIT scores.

Table I. Characteristics and drinking patterns independently associated with high-risk drinking in current drinkers*

\begin{tabular}{|c|c|c|c|c|}
\hline & \multicolumn{2}{|c|}{ Gauteng $(N=152)$} & \multicolumn{2}{|c|}{ Western Cape $(N=170)$} \\
\hline & OR $(95 \%$ CI) & $p$-value & OR $(95 \%$ CI $)$ & $p$-value ${ }^{\dagger}$ \\
\hline Currently employed & $0.3(0.1-0.8)$ & 0.008 & - & - \\
\hline Possessing 5 or more household amenities & - & - & $0.4(0.2-1.1)$ & 0.069 \\
\hline Household never/seldom goes hungry & $0.3(0.1-0.9)$ & 0.030 & $0.2(0.3-1.2)$ & 0.079 \\
\hline \multicolumn{5}{|l|}{ Presence of alcohol problems in at least } \\
\hline 1 family member & $6.0(2.3-15.7)$ & $<0.001$ & $3.1(1.5-6.4)$ & 0.002 \\
\hline Current smoker & - & - & $7.6(3.1-18.9)$ & $<0.001$ \\
\hline \multicolumn{5}{|c|}{$\begin{array}{l}{ }^{*} \text { Multiple logistic regression. Variables originally entered in model: age at interview, marital status, proxies of SES, presence of alcohol problems in the family, age at onset of alcohol } \\
\text { consumption and current smoking. All CIs adjusted for clustering. }\end{array}$} \\
\hline \multicolumn{5}{|c|}{${ }^{+}$Some $p$-values initially $<0.05$ became $>0.05$ after adjusting for clustering. } \\
\hline
\end{tabular}


(Western Cape) and respondents with a family member with an alcohol problem (both sites) were more likely to be high-risk drinkers (Table I).

\section{Discussion}

This study highlights the extent and characteristics of alcohol consumption in women of reproductive age, at an urban site in Gauteng and a rural site in the Western Cape. A large proportion of the women in these areas do not consume alcohol, but a significant proportion of those who consume alcohol do so at high-risk levels, especially in the Western Cape.

Measures of low socio-economic status (SES) and an alcohol problem in one or more family members were associated with high-risk drinking in both sites, which is consistent with the literature. ${ }^{6,7}$ Having parents, siblings or partners who abuse alcohol fosters an environment where alcohol consumption is modelled, accepted and encouraged.

Current smoking was associated with high-risk drinking for the Western Cape. Alcohol and tobacco are often used concurrently, therefore suggesting that these form a cluster of habits that can be addressed concomitantly by health programmes.

Increased risk of alcohol-exposed pregnancy, ${ }^{9}$ accidents and violence ${ }^{10}$ are negative effects of high-risk drinking by women. Interventions are therefore urgently needed to reduce the prevalence of high-risk drinking in women. Tailored interventions for families with existing alcohol problems may prevent the cycle of intergenerational and intra-family risky drinking. Women of lower SES, and women who currently smoke, may also benefit from screening and brief interventions in the clinical setting. ${ }^{11}$ Further research into high-risk drinking among women, and prevention strategies, are urgently required in South Africa.

This study has some limitations. Results were based on cross-sectional data, which makes it difficult to determine temporal relationships between risk factors and outcomes. Sub-strata sample sizes were small, with limited power to detect certain associations. Alcohol consumption by women is a sensitive subject that may lead to under-reporting. Although the Gauteng site is likely to resemble other urban areas, the Western Cape site probably differs from other rural nonwine farming areas in the country. Results must therefore be generalised to other rural areas with caution.

This survey was supported by Cooperative Agreement Number 1 U01 DD00044 from the Center for Disease Control \& Prevention (CDC), USA. Its content is solely the responsibility of the authors and does not necessarily represent the official views of the CDC.

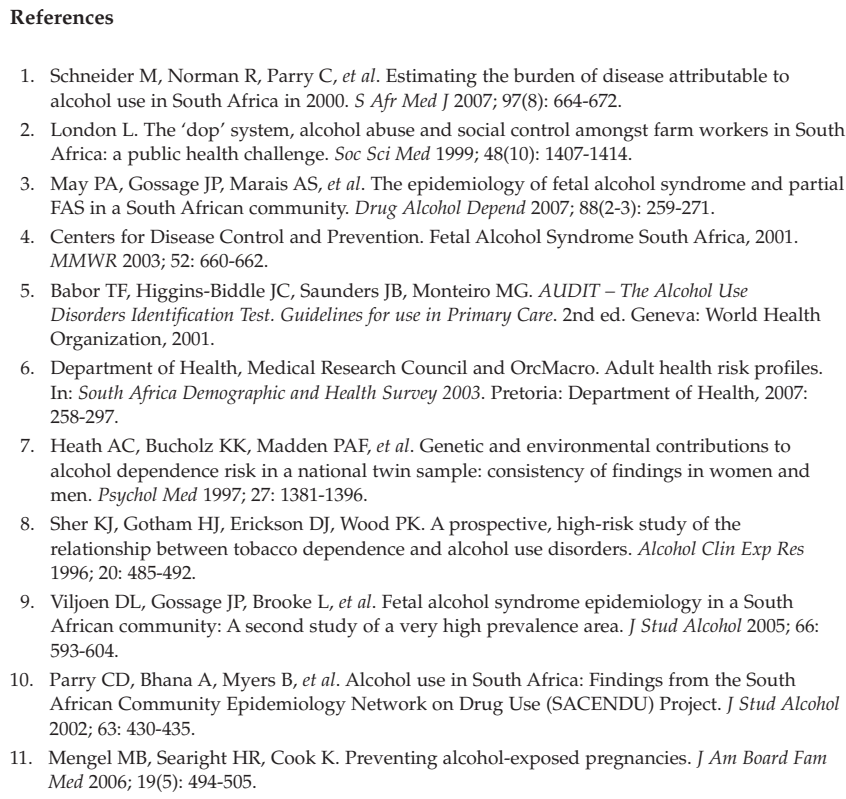

5. Babor TF, Higgins-Biddle JC, Saunders JB, Monteiro MG. AUDIT - The Alcohol Use Babor TE, Higgins-Biddle JC, Saunders JB, Monteiro MG. AUDIT - The Alcohol Use
Disorders Identification Test. Guidelines for use in Primary Care. 2nd ed. Geneva: World Health Disorders Identificatio

6. Department of Health, Medical Research Council and OrcMacro. Adult health risk profiles. In: South Africa Demographic and Health Survey 2003. Pretoria: Department of Health, 2007: 258-297

7. Heath AC, Bucholz KK, Madden PAF, et al. Genetic and environmental contributions to alcohol dependence risk in a national twin sample: consistency of findings in women and men. Psychol Med 1997; 27: 1381-1396.

8. Sher KJ, Gotham HJ, Erickson DJ, Wood PK. A prospective, high-risk study of the relationship between tobacco dependence and alcohol use disorders. Alcohol Clin Exp Res 1996; 20: 485-492.

9. Viljoen DL, Gossage JP, Brooke L, et al. Fetal alcohol syndrome epidemiology in a South African community: A second study of a very high prevalence area. J Stud Alcohol 2005; 66: 593-604.

10. Parry CD, Bhana A, Myers B, et al. Alcohol use in South Africa: Findings from the South African Community Epidemiology Network on Drug Use (SACENDU) Project. J Stud Alcohol 2002; 63: 430-435.

11. Mengel MB, Searight HR, Cook K. Preventing alcohol-exposed pregnancies. J Am Board Fam Med 2006; 19(5): 494-505.

Accepted 27 November 2009. 